\title{
THE PLANAR CLOSING LEMMA FOR CHAIN RECURRENCE
}

\author{
MARIA LUCIA ALVARENGA PEIXOTO AND CHARLES CHAPMAN PUGH
}

ABSTRACT. If $z$ is a chain recurrent point of a $C^{r}$ planar flow, all of whose fixed points are hyperbolic, then it is proved that the orbit through $z$ becomes periodic under a perturbation that is $C^{r}$ small in the Whitney topology.

\section{INTRODUCTION}

Let $\varphi$ be a flow on $M$, a metric space. The forward orbit of $p \in M$ under $\varphi$ is

$$
O_{+}(p)=\left\{\varphi_{t} p: t \geq 0\right\} .
$$

There are several natural ways to extend $O_{+}, \varphi$-invariantly. The simplest is to form

$$
\overline{O_{+}}(p)=O_{+}(p) \cup \omega(p)
$$

where $\omega(p)$ is the $\omega$-limit set of $p$,

$$
\omega(p)=\left\{x \in M: \varphi_{t_{n}}(p) \rightarrow x \text { for some } t_{n} \rightarrow \infty\right\} .
$$

Thus, $\overline{O_{+}}(p)$ is the closure of $O_{+}(p)$. Although $\omega(p)$ is closed and $\varphi-$ invariant, there may be points $q$ near it which tend to new limits. This leads one to define the first prolongation of $O_{+}(p)$ as

$$
\begin{aligned}
& P_{1}(p)=O_{+}(p) \cup J_{1}(p), \\
& J_{1}(p)=\left\{y \in M: \varphi_{t_{n}}\left(x_{n}\right) \rightarrow y \text { for some } t_{n} \rightarrow \infty \text { and } x_{n} \rightarrow x\right\} .
\end{aligned}
$$

Repeating the process transfinitely, with neighborhood closure at limit ordinals, leads to the ultimate prolongation of $O_{+}(p)$ :

$$
P(p)=O_{+}(p) \cup \bigcup_{\alpha} J_{\alpha}(p)=\bigcup_{\alpha}^{\dagger} P_{\alpha}(p)
$$

where $\alpha$ ranges through all ordinals. See Bhatia and Szegő (1970) for details. Correspondingly we write $J(p)=\bigcup_{\alpha} J_{\alpha}(p)$. If $M$ is separable then it turns out that some countable ordinal $\beta=\beta(\varphi)$ suffices, that is

$$
P_{\alpha}(p)=P_{\beta}(p) \text { for all } \alpha \geq \beta \text {. }
$$

Surprisingly, a more general extension $O_{+}(p)$ exists based on the concept of $(\varepsilon, T)$-chains.

Received by the editors March 4, 1991 and, in revised form, May 29, 1991.

1991 Mathematics Subject Classification. Primary 58Fxx; Secondary 58F10, 58F30, 34C28, 34D10, 34D30. 
Definitions. An orbit segment of $\varphi$ is a set $\left\{\varphi_{t} x: 0 \leq t \leq \tau\right\}$ for some $\tau \geq 0$. We denote it by $[x, y]$ where $y=\varphi_{\tau}(x)$. Its duration is $\tau$. An $(\varepsilon, T)$-chain from $p$ to $q$ is a finite collection $\Gamma$ of orbit segments $\left[x_{1}, y_{1}\right], \ldots,\left[x_{n}, y_{n}\right]$ each of duration $\geq T$ such that $d\left(y_{i}, x_{i+1}\right) \leq \varepsilon, 0 \leq i \leq n$, where we understand $p=y_{0}$ and $q=x_{n+1}$. See Figure 1.1.

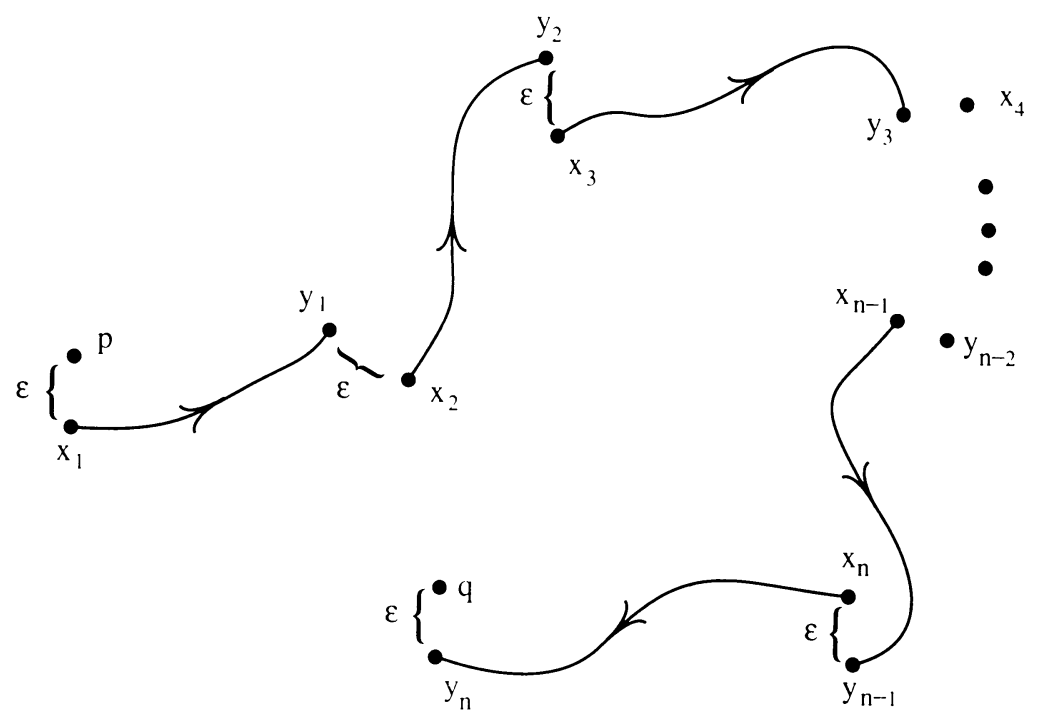

FiguRE 1.1. An $(\varepsilon, T)$-chain from $p$ to $q$.

The chain extension of $O_{+}(p)$ is $O_{+}(p) \cup E_{+}(p)$ where $E_{+}(p)=\{q \in M$ : for all $\varepsilon, T>0$, there exists an $(\varepsilon, T)$-chain from $p$ to $q$ \} .

If $M$ is not compact then the $\varepsilon$ and $T$ above should be thought of as positive continuous functions defined on $M$. They are "local constants". An $(\varepsilon, T)$-chain is a finite collection of orbit segments, as before, satisfying

(a) $\left[x_{i}, y_{i}\right]$ has duration $\geq T\left(x_{i}\right), 1 \leq i \leq n$;

(b) $d\left(y_{i}, x_{i+1}\right)<\varepsilon\left(y_{i}\right), 0 \leq i \leq n$.

Note. Nitecki (1978) requires $T$ to be constant, even when $M$ is noncompact. In Appendix 1, we show that this does not affect the definition of chain extension.

Corresponding to concepts of orbit extension are concepts of recurrence. The point $p \in M$ is

$\begin{array}{ll}\text { fixed } & \text { if }\{p\}=O_{+}(p), \\ \text { periodic } & \text { if } p \in O_{+}(p) \neq\{p\}, \\ \omega \text {-recurrent } & \text { if } p \in \omega(p), \\ \text { nonwandering } & \text { if } p \in J_{1}(p), \\ \text { prolongationally recurrent } & \text { if } p \in \bigcup_{\alpha} J_{\alpha}(p), \\ \text { chain recurrent } & \text { if } p \in E_{+}(p) .\end{array}$

How much do these recurrences coincide? The Closing Lemma may be viewed as an attempt to force coincidence by approximation: $\omega$-recurrent 
points and $J_{1}$ recurrent points become periodic after a perturbation of the dynamics. See Pugh (1967a, and b) respectively. The Closing Lemma for more general recurrence has not met with success, although in Pugh (1984), an example is given to show that the $J_{2}$ Closing Lemma is false on noncompact surfaces, and in Pugh (1975) there is an example against $C^{2}$-double closing of $J_{2}$-recurrence on the 2-torus. Here we verify the Closing Lemma for some planar chain recurrence.

1.1. Theorem. Suppose $X$ is a $C^{r}$ vectorfield on an open subset $M$ of $S^{2}$, $1 \leq r \leq \infty, X$ generates a flow $\varphi$, and $z \in M$ is chain recurrent. Suppose all the fixed points of $\varphi$ are hyperbolic. If $\mathscr{U}$ is a neighborhood of $X$ in the $C^{r}$ Whitney topology then $\mathscr{U}$ contains a field $Y$ for which $z$ is periodic.

See $\S 5$ for the proof of this theorem.

Remarks. $S^{2}$ minus a point is $R^{2}$ so the theorem applies to flows on $R^{2}$. The assumption about hyperbolic fixed points reflects our inability to solve the following.

Local singular connecting problem. Let $p$ be an isolated fixed point of the smooth flow $\varphi$ on $R^{2}$. Suppose $\omega(x)=p=\alpha(y)$ for some points $x, y \neq p$.

Can $\varphi$ be $C^{r}$ perturbed to put $x, y$ on the same trajectory?
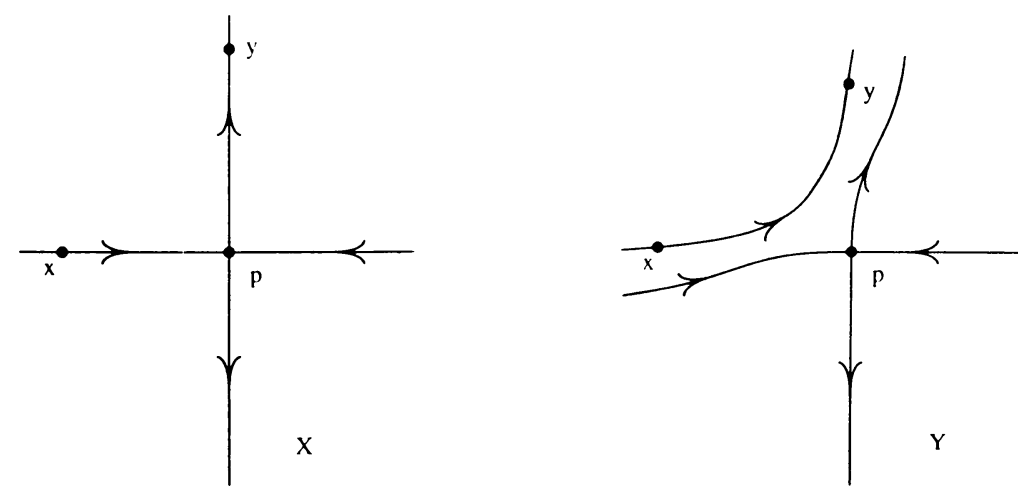

Figure 1.2. Connecting $x$ to $y$ via $p$ in the hyperbolic case; $Y$ approximates $X=\dot{\varphi}$.
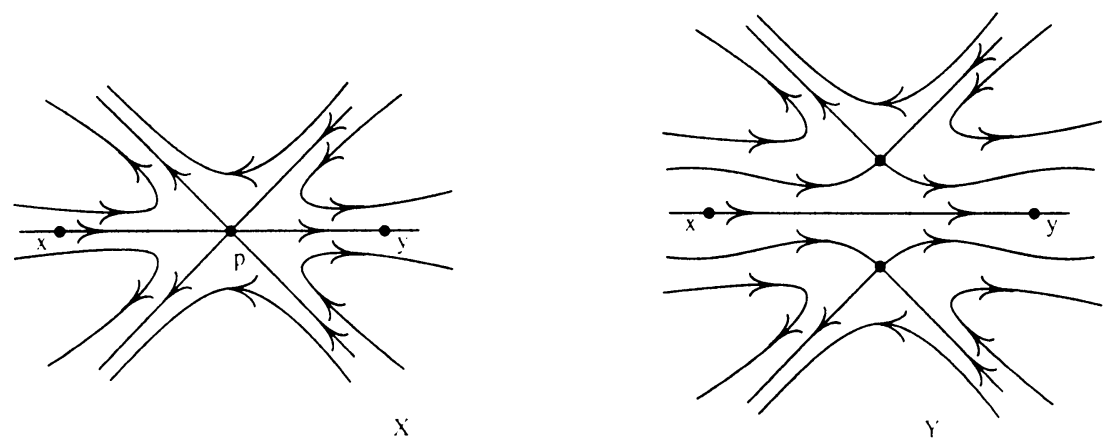

Figure 1.3. Connecting $x$ to $y$ via $p$ in a nonhyperbolic case; $Y$ approximates $X=\dot{\varphi}$. 
If $p$ is hyperbolic then the answer is "yes"; see Figure 1.2. In some other cases (Figure 1.3) the answer is also "yes", but in general the problem may be hard.

\section{THE WHITNEY TOPOLOGY}

If $M$ is a compact manifold, it is natural to topologize the space of $C^{r}$ tangent vector fields on $M, \mathscr{X}^{r}(M)$, with the $C^{r}$-uniform topology. When $M$ is no longer compact, such as $M=\mathbf{R}^{2}$, one uses the Whitney topology. It can be defined in several equivalent ways, one of which is the following. Note that the definition we give is simpler than the usual one which involves coordinate coverings.

First take the case that $M$ is Euclidean space, $M=\mathbf{R}^{n}$. If $\varepsilon$ is a positive continuous function on $\mathbf{R}^{n}$ and $X \in \mathscr{X}^{r}\left(\mathbf{R}^{n}\right)$ then we set

$$
\begin{aligned}
\mathscr{N}_{\varepsilon}(X)=\left\{Y \in \mathscr{X}^{r}\left(\mathbf{R}^{n}\right): \text { for all } x \in \mathbf{R}^{n},\right. \\
\\
\left.\qquad|Y(x)-X(x)|+\cdots+\left\|D^{r}(Y-X)_{x}\right\|<\varepsilon(x)\right\} .
\end{aligned}
$$

These $\mathscr{N}_{\varepsilon}(X)$ are a neighborhood base at $X$ for the $C^{r}$ Whitney topology on $\mathscr{X}^{r}\left(\mathbf{R}^{n}\right)$.

Next take the case that $M$ is a general smooth $m$-manifold. The meaning of $\left\|D^{r}(Y-X)\right\|$ becomes a problem which we bypass by choosing

(a) a proper smooth embedding $M^{m} \hookrightarrow \mathbf{R}^{n}$,

(b) the proper smooth tubular neighborhood retraction $\rho: N \rightarrow M$ along short normal segments to $M$ in $\mathbf{R}^{n}$,

(c) a smooth bump function $\beta: N \rightarrow[0,1]$ which is identically zero near $\partial N$ and identically one near $M$.

Then we define the extension operator $e: \mathscr{X}^{r}(M) \rightarrow \mathscr{X}^{r}\left(\mathbf{R}^{n}\right)$ by

$$
e(X)= \begin{cases}\beta(x) \rho^{*} X(x), & x \in N, \\ 0, & x \notin N,\end{cases}
$$

where $\rho^{*} X$ is the parallel extension of $X$ to $N$ along $\rho$-fibers. That is, $\rho^{*} X(x) \in T_{x}\left(\mathbf{R}^{n}\right)$ is the $\mathbf{R}^{n}$-parallel-translate of $X(\rho(x))$.

The Whitney topology on $\mathscr{X}^{r}(M)$ is the $e$-pullback of that on $\mathscr{X}^{r}\left(\mathbf{R}^{n}\right)$. That is, a neighborhood base at $X \in \mathscr{X}^{r}(M)$ is given by $\left\{Y \in \mathscr{X}^{r}(M): e(Y) \in\right.$ $\left.\mathscr{N}_{\varepsilon}(e X)\right\}$ where $\varepsilon$ runs through all positive continuous functions on $\mathbf{R}^{n}$. It is independent of the choices made in (a), (b), (c).

2.1. Convexity Proposition. $\mathscr{Z}^{r}(M)$ has a neighborhood base $\mathscr{V}$ with the following property. If $V$ is a $\mathscr{V}$ neighborhood of $X$, if $\Delta_{1}, \Delta_{2}, \ldots, \Delta_{n}$ in $\mathscr{X}^{r}(M)$ have disjoint supports, and if $Y_{i}=X+\Delta_{i}$ lies in $V$ for each $i$ then $Y=X+\Sigma \Delta_{i}$ also lies in $V$.

Proof. Suppose $M=\mathbf{R}^{n}$. Since $\mathscr{N}_{\varepsilon}(X)$ is defined pointwise, it is clear that it has the above property. If $M \hookrightarrow \mathbf{R}^{n}$ then $\mathscr{X}^{r}(M)$ inherits this property from $\mathscr{X}^{r}\left(\mathbf{R}^{n}\right)$ under $e$-pullback.

\section{FlowboXeS AND LIFT}

To quantify the perturbations we use for the Closing Lemma, we introduce flowboxes in a uniform way. 
Let $\varphi$ be a $C^{r}$ flow on a manifold $M^{m}$. We assume $M$ equipped with some fixed smooth Riemann structure. As standing notation we call $M_{0}$ the set of regular points of $\varphi$,

$$
M_{0}=\{x \in M: x \text { is not a fixed point of } \varphi\} .
$$

Choose and fix a smooth $(m-1)$ plane bundle over $M_{0}$ complementary to $X$,

$$
\Pi \oplus \operatorname{span}(X)=T M_{0} .
$$

For any $x \in M_{0}$, there exists $\nu>0$ such that the map

$$
f_{x}^{\nu}:[-\nu, \nu] \times \Pi_{x}(\nu) \rightarrow M, \quad(t, v) \rightarrow \varphi_{t}\left(\exp _{x}(v)\right)
$$

is a $C^{r}$ embedding, where $\Pi_{x}(\nu)$ is the disc of radius $\nu$ in $\Pi_{x}$. Since $M$ is locally compact we may determine some positive continuous function $\nu$ on $M_{0}$ such that $f_{x}^{\nu(x)}$ is an embedding for each $x \in M_{0}$. Fix such a flowbox-radius function $\nu$.

Definition. The canonical flowbox of radius $\nu(x)$ at $x \in M_{0}$ is the range $F_{x}^{\nu}$ of the chart map $f_{x}^{\nu(x)}$. If $0<c \leq 1$ then the c-central portion of $F_{x}^{\nu}$ is the set

$$
\left\{\varphi_{t}\left(\exp _{x}(v)\right):|t| \leq \nu(x) \text { and } v \in \Pi_{x}(c \nu(x))\right\} .
$$

3.1. Lift Lemma. Let $\mathscr{U}$ be a neighborhood of $X=\dot{\varphi}$ in the $C^{r}$ Whitney topology on $\mathscr{X}^{r}(M), r \geq 1$, and let $\mu: M_{0} \rightarrow R$ be a continuous function such that $0<\mu(x)<\nu(x)$ for all $x \in M_{0}$. Then there exists a positive continuous function $c$ on $M_{0}$ such that if $y, y^{\prime}$ lie at opposite ends of the $c(x)$-central portion of the canonical $F_{x}^{\nu}$ then there exists a field $Y \cdot \in \mathscr{U}$ with $Y \equiv X$ off $F_{x}^{\mu}$ and, under the $Y$-flow, $y$ flows to $x$ which flows to $y^{\prime}$ across the $c(x)$-central portion of $F_{x}^{\nu}$.

Proof. Consider Figure 3.1.

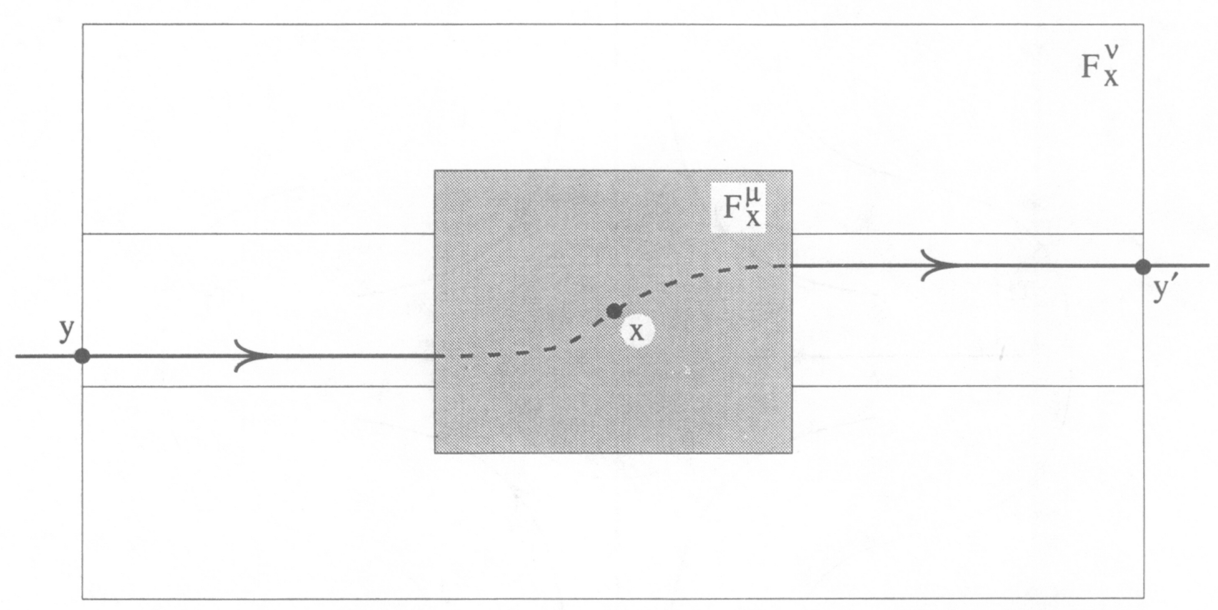

FIGURE 3.1. $Y$ lifts $y$ to $x$ and $x$ to $y^{\prime}$.

\section{QUASIORBITS}

A $C^{1}$ curve $\lambda(t)$ in $M$ is an $\varepsilon$-quasiorbit of $X$ if $\left|\lambda^{\prime}(t)-X(\lambda(t))\right|<\varepsilon$ for all $t$ where $\lambda$ is defined. In other words, it is an $\varepsilon$-approximate solution 
of the differential equation $x^{\prime}=X(x)$. When $M$ is noncompact it is natural to replace the constant $\varepsilon$ with a positive continuous function $\varepsilon(x)$. Then we require $\left|\lambda^{\prime}(t)-X(\lambda(t))\right|<\varepsilon(\lambda(t))$.

In Nitecki (1978) it is shown that a point $z$ is chain recurrent if and only if for every positive continuous function $\varepsilon>0$ on $M$ there exists a periodic $\varepsilon$-quasiorbit through $z$.

4.1. Global Replacement Lemma. Suppose that the fixed points of the $X$-flow on $M^{2}$ are hyperbolic, therefore countable in number, say $p_{1}, p_{2}, \ldots$ and $z$ is a chain recurrent point, $z \in M_{0}$. Then there is a neighborhood $V$ of $\left\{p_{i}\right\}$ such that if $\varepsilon: M \rightarrow[0, \infty)$ is a given continuous function, positive on $M \backslash \bar{V}$, then there is a periodic $\varepsilon$-quasiorbit $\lambda$ through $z$. Moreover $\lambda$ is a simple closed curve.

Remark. We permit $\varepsilon \equiv 0$ on $\bar{V}$. Then $\lambda \cap \bar{V}$ consists of true $X$-trajectory arcs.

4.2. Local Replacement Lemma. Suppose $p$ is a hyperbolic fixed point for a flow $\varphi$ in $\mathbf{R}^{2}$ and $U$ is a neighborhood of $p$ on which $\varphi$ is linearizable. Let $N \subset U$ be a small neighborhood of $p$ and let $T_{ \pm}, T_{ \pm}^{*}$ be transversals to $W^{s}(p), W^{u}(p)$ as shown in Figure 4.1. Then, for every $\varepsilon>0$ there exists $\delta>0$ such that any $\delta$-quasiorbit $\sigma$ from $T$ to $T^{*}$ in $U$ can be replaced by an $\varepsilon$-quasiorbit $\lambda$ from $T$ to $T^{*}$ in $U$ such that
(a) $\lambda=\sigma$ near $T$ and $T^{*}$,
(b) $\lambda \cap N$ is a true orbit arc,
(c) $\lambda$ is an arc.

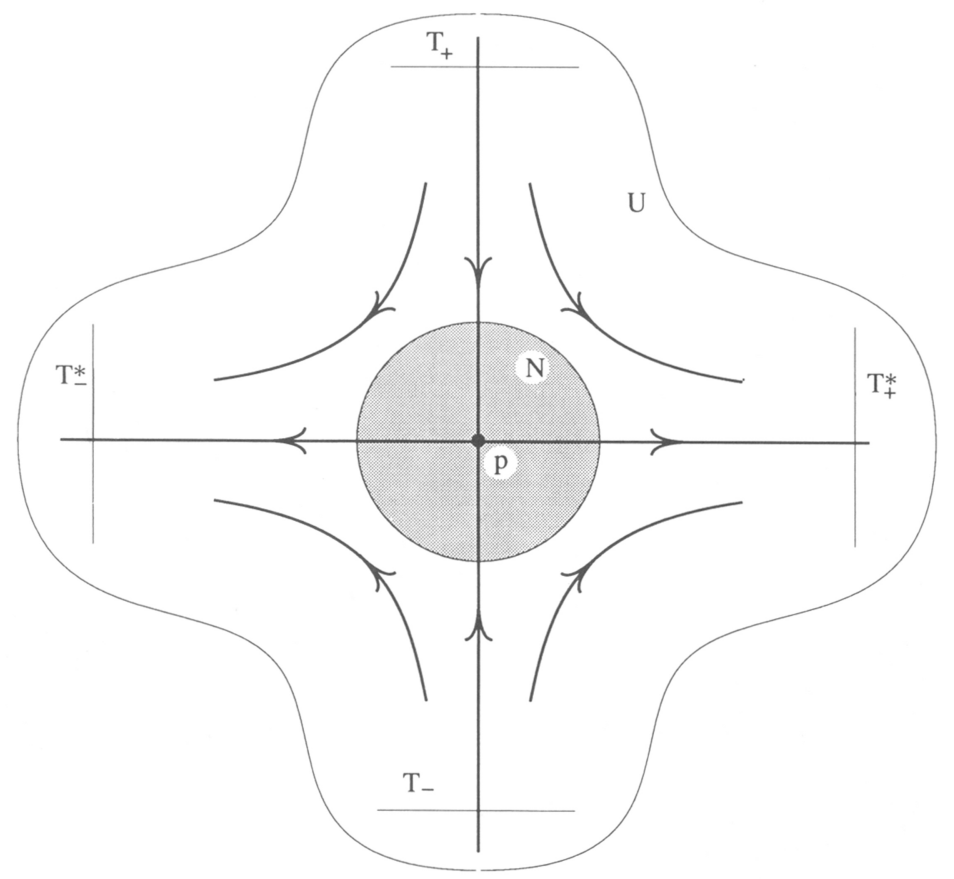

FigURE 4.1 
Remark. This result is valid in dimension $\leq 3$ but not in dimension $\geq 4$. It is easy to see that (c) is implied by (a) and (b).

Proof. We draw flowboxes $F_{ \pm}, F_{ \pm}^{*}$ across $W^{u}(p), W^{s}(p)$ to get the situation shown in Figure 4.2.

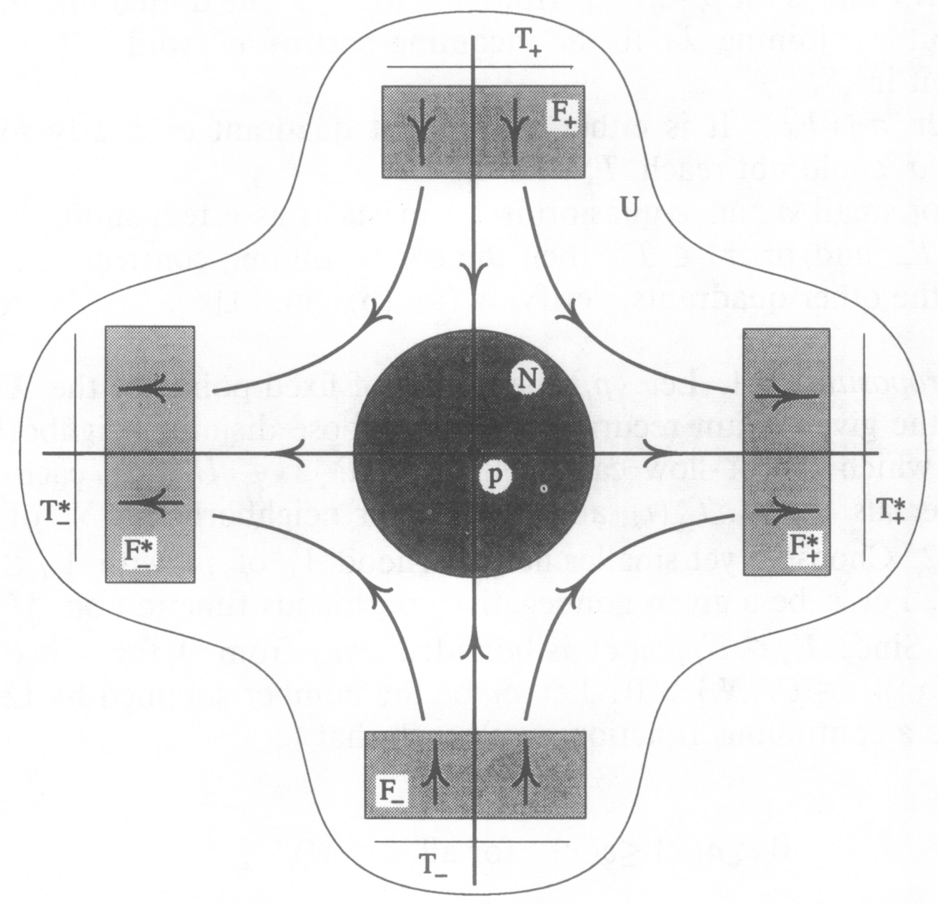

FIGURE 4.2
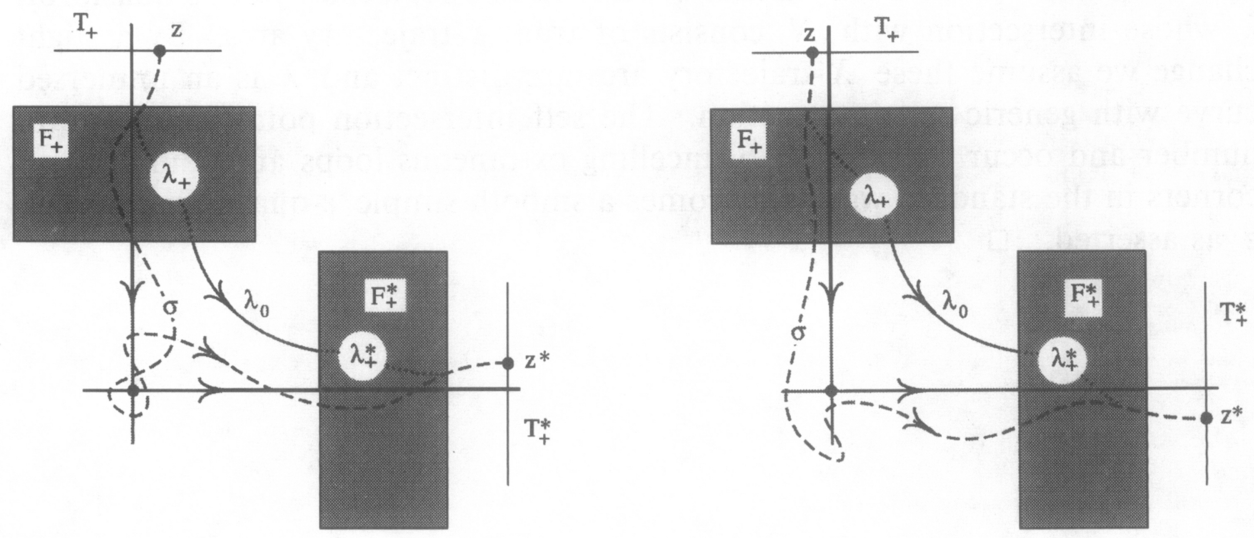

FIGURE 4.3 
Let $\sigma$ be a $\delta$-quasiorbit from $z \in T$ to $z^{*} \in T^{*}$ in $U$. When $\delta$ is small, the parts of $\sigma$ outside $N$ are nearly trajectory arcs in the $C^{1}$ sense. Near $p$, $\sigma$ may be quite twisted, because the angle between $X$ and $\sigma^{\prime}$ may be large when $X$ and $\left|X-\sigma^{\prime}\right|$ are small.

Suppose $z \in T_{+}, z^{*} \in T_{+}^{*}$. According to Appendix 2, we may replace $\sigma$ with a $\varphi$-arc $\lambda_{0}$ from $F_{+}$to $F_{+}^{*}$ (necessarily in the first quadrant) together with $\delta$-quasiorbits across $F_{+}, F_{+}^{*}$ as shown. See Figure 4.3(a). Note that $z, z^{*}, \sigma$ need not lie in the first quadrant. See Figure 4.3(b).

The heavy line is the $\varphi$-arc $\lambda_{0}$ from $F_{+}$to $F_{+}^{*}$. The dotted line in $F_{+}$is an $\varepsilon$-quasiorbit $\lambda_{+}$joining $\lambda_{0}$ to the incoming part of $\sigma$, while $\lambda_{+}^{*}$ is a similar $\varepsilon$-quasiorbit in $F_{+}^{*}$.

Consider $\sigma \cap F_{+}$. It is either in the first quadrant or nearly so, because otherwise $\sigma$ could not reach $T_{+}^{*}$.

Thus, for small $\delta$, an $\varepsilon$-quasiorbit $\lambda_{+}$exists as asserted; similarly $\lambda_{+}^{*}$.

If $z \in T_{-}$and/or $z^{*} \in T_{-}^{*}$ then the corresponding construction places $\lambda_{0}$ in one of the other quadrants, verifying (a), (b), (c).

Proof of Proposition 4.1. Let $\left\{p_{i}\right\}$ be the set of fixed points of the $X$-flow and $z_{0} \in M_{0}$ the given chain recurrent point. Choose disjoint neighborhoods $U_{i}$ of $p_{i}$ on which the $X$-flow can be linearized, $z \notin U_{i}$. In each $U_{i}$ draw the transversals $T_{ \pm}(i), T_{ \pm}^{*}(i)$ and the smaller neighborhood $N_{i}$ of $p_{i}$ as in Lemma 4.2. Choose a yet smaller neighborhood $V_{i}$ of $p_{i}$ with $\bar{V}_{i} \subset N_{i}$. Call $V=\bigcup_{i} V_{i}$. Let $\varepsilon$ be a given nonnegative continuous function on $M$, positive on $M \backslash \bar{V}$. Since $\bar{V}_{i} \subset N_{i}, \varepsilon(x)$ is bounded away from 0 for $x \in U_{i} \backslash N_{i}$; say $\varepsilon_{i}=\inf \left\{\varepsilon(x): x \in U_{i} \backslash N_{i}\right\}>0$. Let $\delta_{i}$ be the number supplied by Lemma 4.2 and choose a continuous function $\delta(x)$ such that

$$
\begin{aligned}
0<\delta(x) \leq \varepsilon(x) & \text { for all } x \in M \backslash U, \\
\delta(x) \leq \delta_{i} & \text { if } x \in U_{i}, U=\bigcup_{i} U_{i} .
\end{aligned}
$$

According to Nitecki's Lemma there is a periodic $\delta$-quasiorbit $\sigma$ through the chain recurrent point $z$. By Lemma 4.2 it can be replaced by an $\varepsilon$-quasiorbit $\lambda$ whose intersection with $N$ consists of true $X$-trajectory arcs. By a slight change we assume these $X$-trajectory arcs are distinct and $\lambda$ is an immersed curve with generic self intersection. The self intersection points are finite in number and occur outside $N$. Cancelling extraneous loops and rounding off corners in the standard way, $\lambda$ becomes a smooth simple $\varepsilon$-quasiorbit through $z$ as asserted. 


\section{TWO-DIMENSIONAL CLOSING}

Before starting the proof of the Closing Lemma, we need two more preliminary results. The first refers to the flowbox radius function $\nu: M_{0} \rightarrow$ $(0, \infty), M_{0}$ being the set of nonfixed points of the $X$-flow. It was constructed in $\S 3$.

5.1. Lemma. There exist positive continuous functions $\varepsilon_{0}, \omega, \mu: M_{0} \rightarrow(0,1)$ such that $0<\mu<\omega<\nu$ and, writing $F_{x}^{\omega}$ for $F_{x}^{\omega(x)}$, etc.,

(a) if $F_{x^{\prime}}^{\omega} \cap F_{x}^{\omega} \neq \varnothing$ then $F_{x^{\prime}}^{\omega} \subset F_{x}^{\nu}$;

(b) if $x^{\prime} \notin F_{x}^{\omega}$ then $F_{x^{\prime}}^{\mu} \cap F_{x}^{\mu}=\varnothing$;

(c) suppose $F_{x_{1}}^{\omega}, F_{x_{2}}^{\omega}$ are contained in $F_{x}^{\nu}$ and $F_{x_{3}}^{\mu}$ meets the convex hull of $F_{x_{1}}^{\omega} \cup F_{x_{2}}^{\omega}$ in $F_{x}^{\nu}$. Then any $\varepsilon_{0}$-quasiorbit $\sigma$ through a point of $F_{x_{3}}^{\mu}$ stretches all the way across $F_{x}^{\nu}$.

Proof. If $\omega \ll \nu$ then intersections of $\omega$-sized boxes occur only near the centers of $\nu$-sized boxes, giving (a). If $\mu \ll \omega$ and $x^{\prime} \notin F_{x}^{\omega}$ then $F_{x^{\prime}}^{\mu}$ is so small that it cannot meet $F_{x}^{\mu}$, giving (b). Since $F_{x}^{\nu}$ is compact, $\omega$ is bounded away from zero on it, and the centers $x_{1}, x_{2}$ of $\omega$-sized boxes are bounded away from $\partial F_{x}^{\nu}$. If $\mu \ll \omega$ then their convex hull $H$ and any $F_{x_{3}}^{\mu}$ meeting $H$ are also bounded away from $\partial F_{x}^{\nu}$. For small enough $\varepsilon_{0}$ this gives (c). See Figure 5.1. Note that (c) applies to all $F_{x_{3}}^{\mu}$ which meet $F_{x}^{\omega}$.

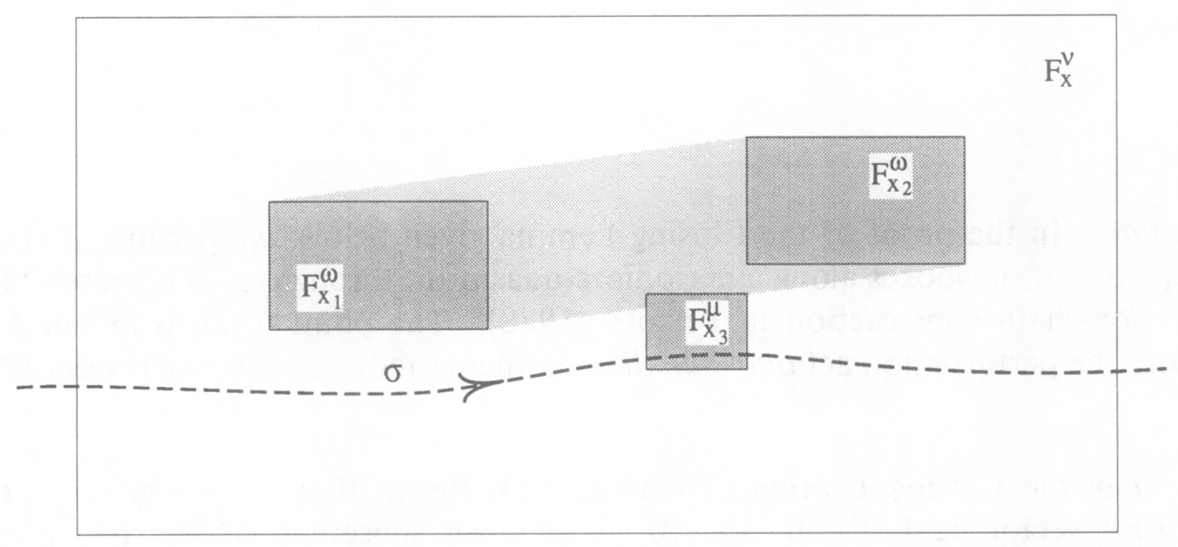

FIGURE 5.1

5.2. Lemma. A simple loop in the plane or sphere which crosses an arc in one direction only, crosses the arc at most once.

Proof. Consider the Poincaré-Bendixson picture in Figure 5.2. 


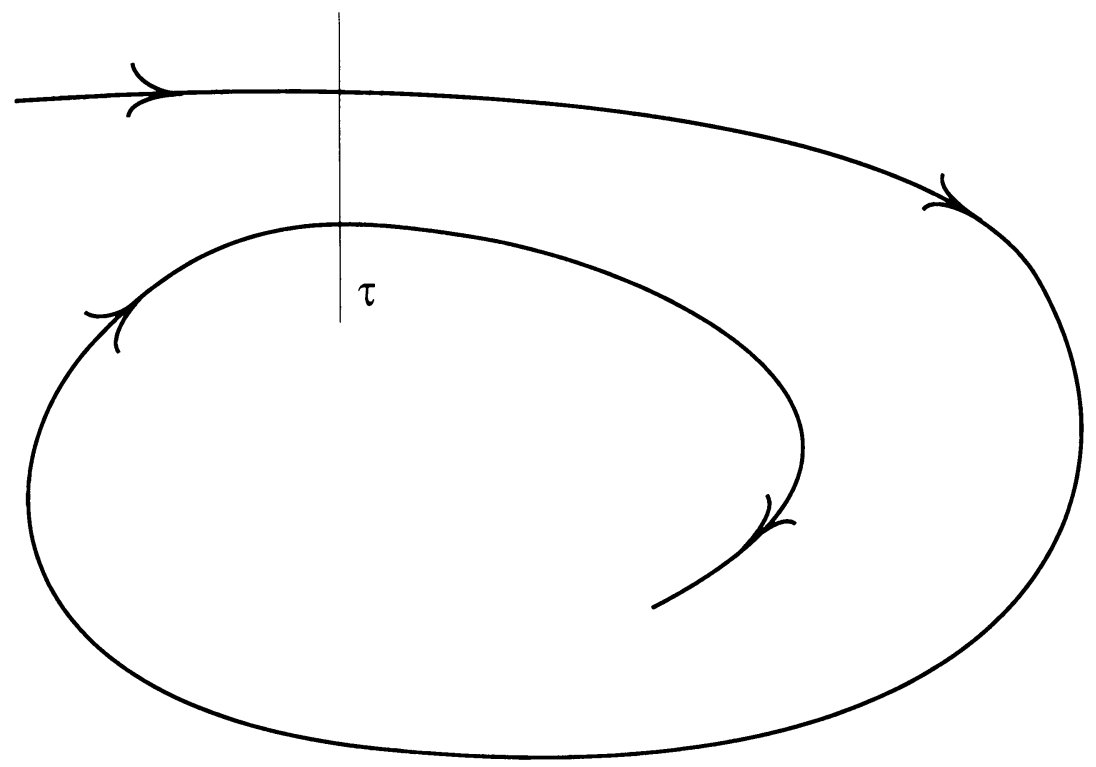

FIGURE 5.2

Remark. In the proof of the Closing Lemma given below, we perturb $X$ in a sequence of flowboxes along a periodic $\varepsilon$-quasiorbit through $z$. This resembles the box-chain construction in Peixoto (1988). The point to keep in mind is "does the perturbation act only one time on the periodic orbit being produced?"

Proof of the Closing Lemma (Theorem 1.1). Recall that we are given a $C^{r}$ tangent vector field $X$ on $M, M$ is an open subset of $S^{2}, \mathscr{U}$ is a given neighborhood of $X$ in the $C^{r}$ Whitney topology, $r \geq 1$, and $z$ is a nonfixed chain recurrent point of the $X$-flow. The fixed points of $X$ are assumed to be hyperbolic. We want to find $Y \in \mathscr{U}$ with a periodic orbit through $z$.

Step 1. By the construction in $\S 3$ we determine the flowbox radius function $\nu$. It is positive and continuous on $M_{0}$, the set of nonfixed points.

Step 2. By Lemma 5.1 we determine positive continuous functions $\omega, \mu, \varepsilon_{0}$ on $M_{0}$.

Step 3. From hyperbolicity of the fixed points we use Proposition 4.1 to determine a suitably small neighborhood $V$ of the fixed point set.

Step 4. From $\mathscr{U}, \mu, \omega$ we use the Lift Lemma 3.1 to determine a small enough $c: M_{0} \rightarrow(0,1]$. Besides, we require $c$ to be so small that on the $c$-central portion of any $F_{x}^{\nu}, c F_{x}^{\nu}$, with $x \in M_{0}$, the following property holds:

(d) If $x^{\prime} \in c F_{x}^{\nu}$ and $F_{x^{\prime}}^{\mu} \subset F_{x}^{\nu}$ then $c F_{x}^{\nu}$ cuts across the middle of $F_{x^{\prime}}^{\mu}$. See Figure 5.3. 


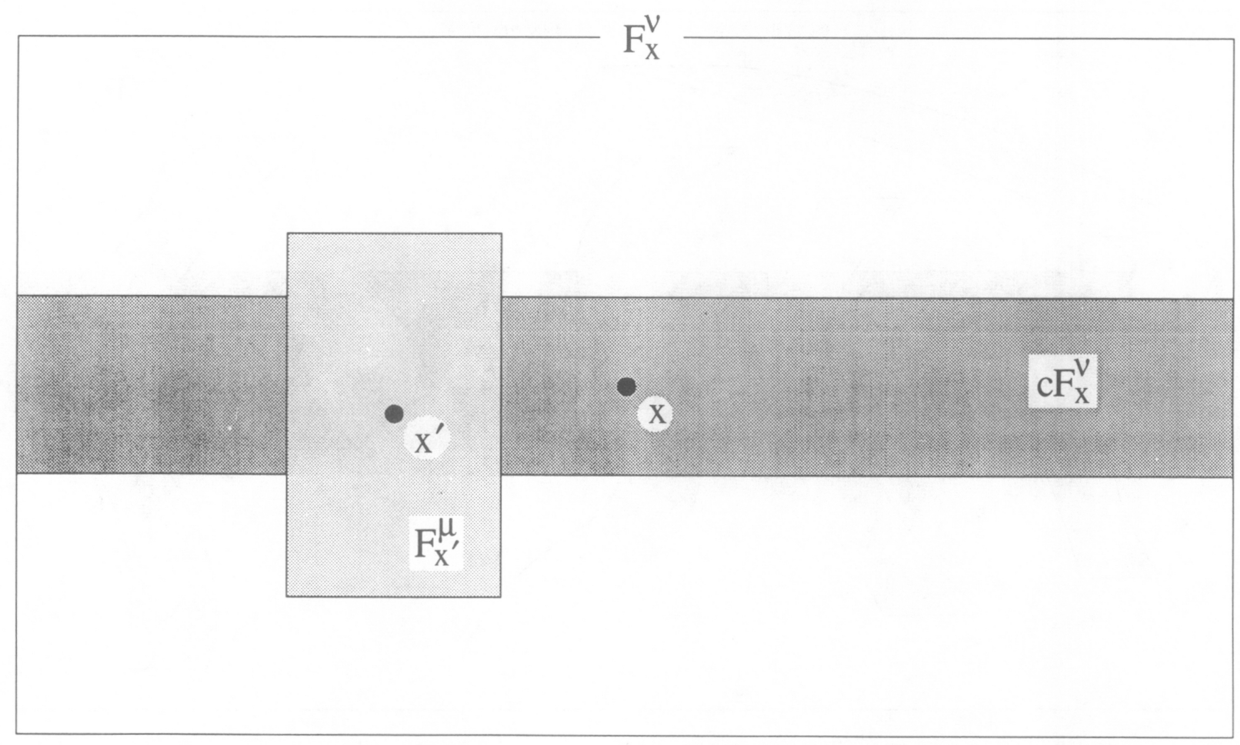

FIGURE 5.3. The $c$-central portion of $F_{x}^{\nu}$ cuts across the middle of $F_{x^{\prime}}^{\mu}$.

It follows from (d) that

(e) If $F_{x_{1}}^{\mu}, \ldots, F_{x_{k}}^{\mu}$ are disjoint, contained in $F_{x}^{\nu}$ and their centers $x_{1}, \ldots$, $x_{k}$ lie in $c F_{x}^{\nu}$, then $c F_{x}^{\nu} \backslash \bigcup F_{x_{i}}^{\mu}$ consists of $k+1$ topological rectangles as shown shaded in Figure 5.4.

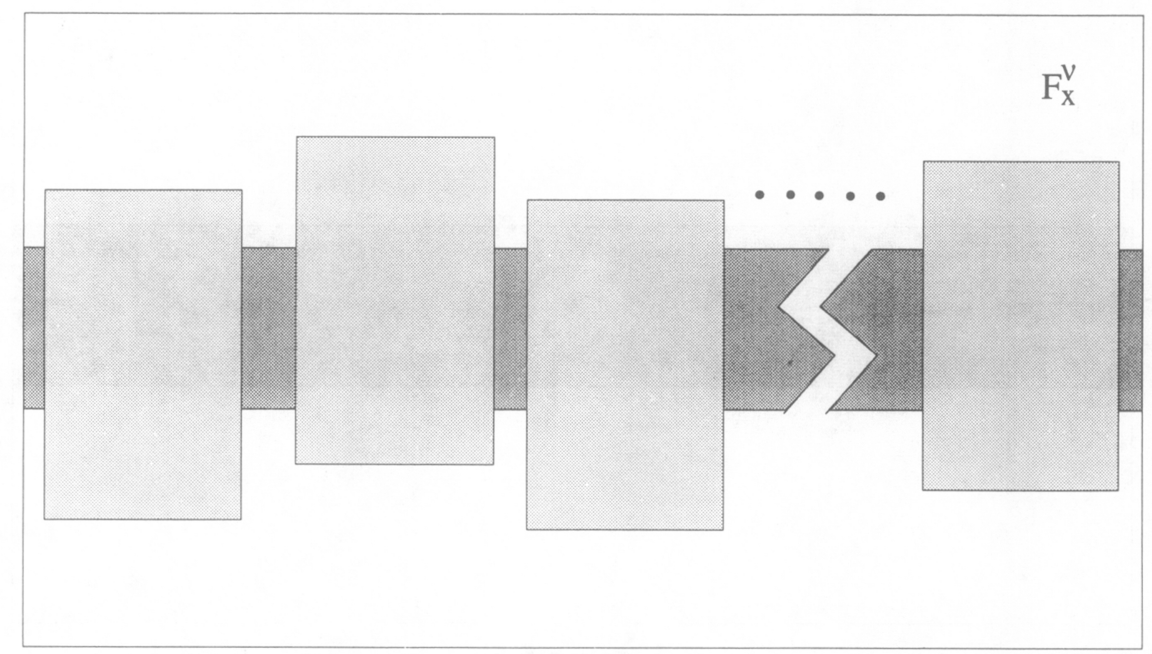

FIGURE 5.4

Note that the actual shape of the $F_{x_{i}}^{\mu}$ can be somewhat contorted, as in Figure 5.5 , but the monotonicity of the shaded rectangles persists. 


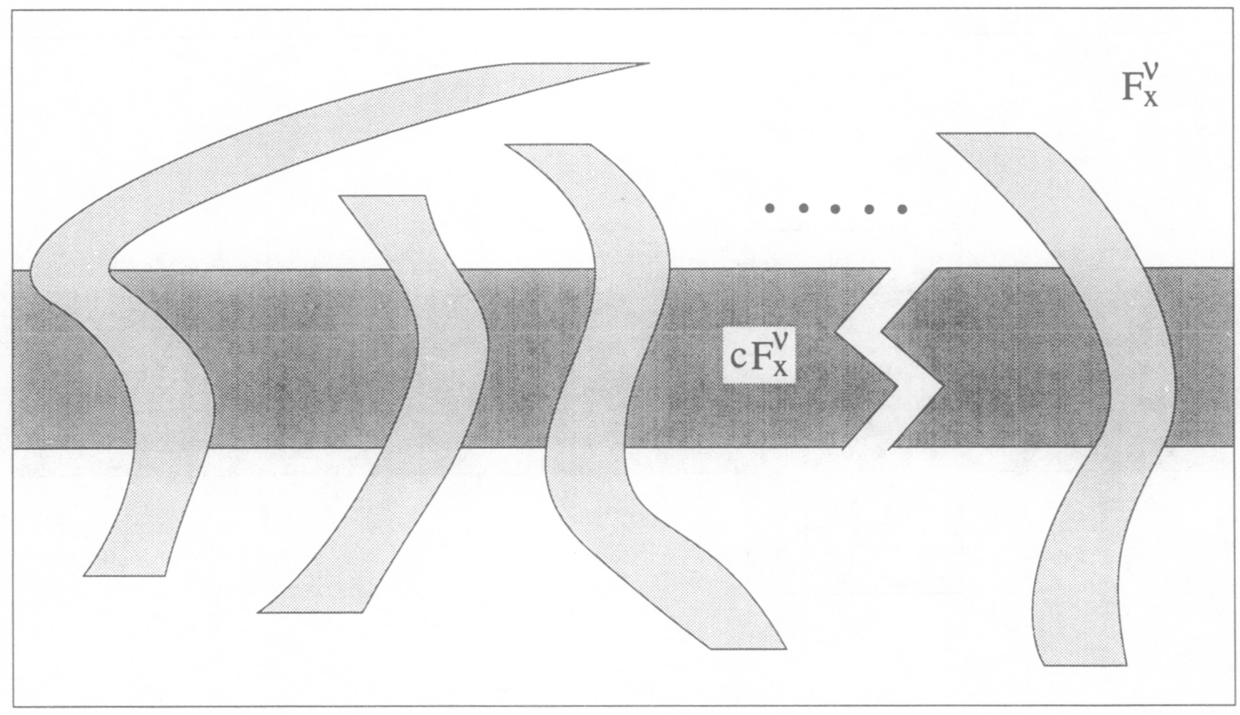

Figure 5.5. Possible shapes of $F_{x_{i}}^{\mu}$.

Step 5. Choose $\varepsilon: M \rightarrow[0,1]$ as follows. We require that $\varepsilon$ is continuous, identically zero on $\bar{V}$, positive off $\bar{V}$, and $\varepsilon \leq \varepsilon_{0}$ everywhere. Also we require $\varepsilon$ to be so small that

(f) If $\sigma$ is any $\varepsilon$-quasiorbit through $x \in M_{0}$, then the connected component of $\sigma \cap F_{x}^{\omega}$ containing $x$ is an arc in the $c$-central portion of $F_{x}^{\omega}$.

It is possible to verify (f) because when $x$ ranges over $M \backslash \bar{V},\left|\sigma^{\prime}-X\right|$ being small implies that the angle between $\sigma^{\prime}$ and $X$ is small, while on $\bar{V}, \varepsilon \equiv 0$. See Figure 5.6.

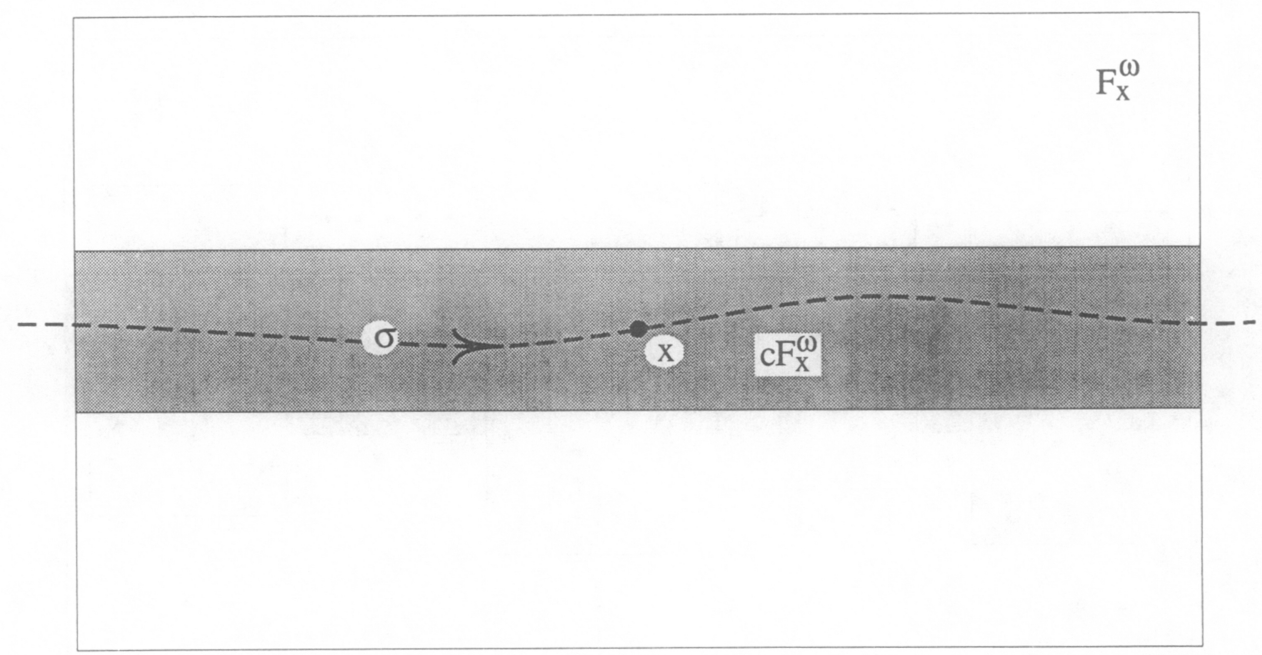

FIGURE 5.6

Note that the $c$-central portion of $F_{x}^{\omega}$ is contained in the $c$-central portion of $F_{x}^{\nu}$.

Step 6. Using this choice of $\varepsilon$ and Proposition 4.1, we determine a smooth simple closed curve $\lambda$ which is a periodic $\varepsilon$-quasiorbit through $z$. In $V, \lambda$ 
consists of true $X$-trajectory arcs. By Lemma 5.2,

(g) If $x \in \lambda$ then $F_{x}^{\mu}, F_{x}^{\omega}, F_{x}^{\nu}$ meet $\lambda$ in nested arcs.

See Figure 5.7. (It is exactly here we use planarity.)

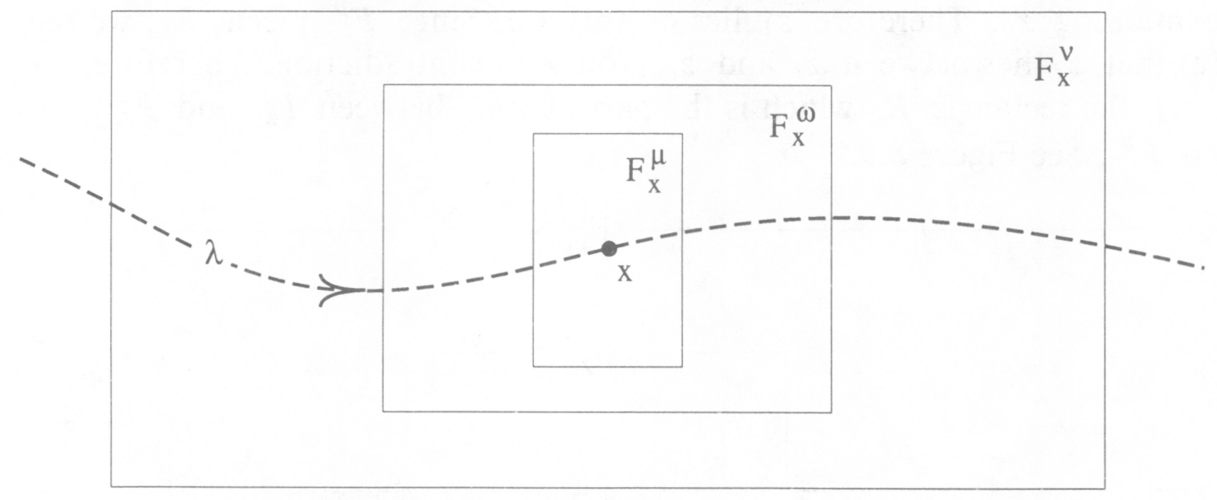

FIGURE 5.7

Step 7. Starting at $z=z_{0}$, we cover $\lambda$ with $\omega$-sized flowboxes as follows. By (f), we know that $\lambda$ exits the right-hand edge of $F_{z_{0}}^{\omega}$ in the $c$-central portion, say at $u_{0}$. Choose $z_{1} \in \lambda$ slightly beyond $u_{0}$ so that $\lambda \cap F_{z_{1}}^{\omega}$ includes $u_{0}$ in its interior. Repeat the construction beyond the point $u_{1}$ where $\lambda \cap F_{z_{1}}^{\omega}$ exits $F_{z_{1}}^{\omega}$. Since $\lambda$ is compact, contained in $M_{0}$ and $\omega$ is continuous, $\omega$ is bounded away from zero on $\lambda$ and we eventually get a finite, indexed cover $F_{z_{0}}^{\omega}, \ldots, F_{z_{n-1}}^{\omega}$ of $\lambda$. We set $z_{n}=z_{0}$ and write indices cyclically. We call $z_{i}$, center points. See Figure 5.8.

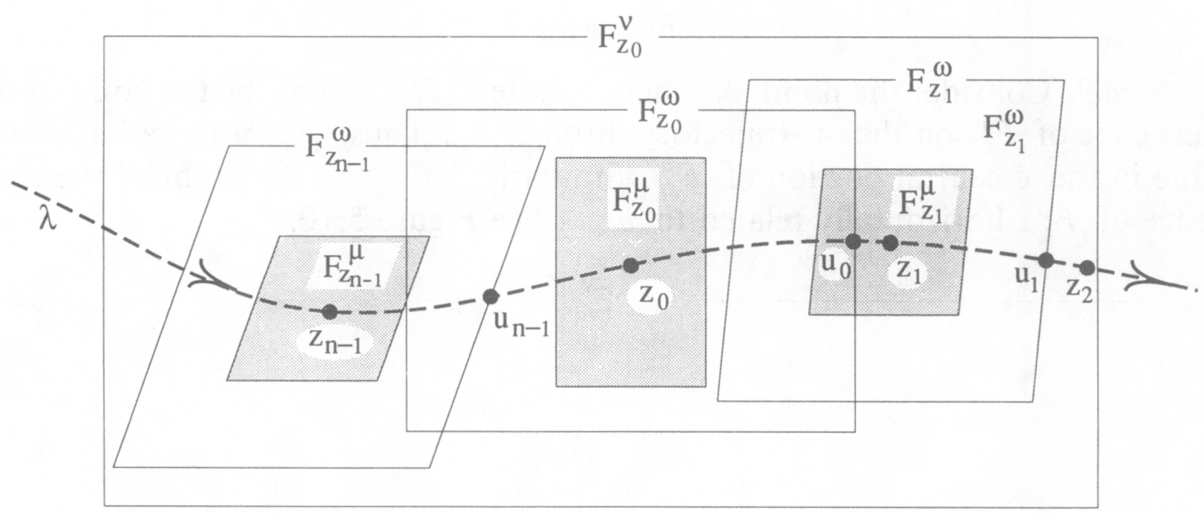

FIGURE 5.8

By $(\mathrm{g})$, choice of $\varepsilon \leq \varepsilon_{0}$, and part (c) of Lemma 5.1, we know that $\lambda_{0}=\lambda \cap F_{z_{0}}^{\nu}$ is an arc crossing all of $F_{z_{0}}^{\nu}$. By (f), $\lambda_{0}$ lies in the $c$-central portion of $F_{z_{0}}^{\nu}$. By part (a) of Lemma 5.1, $F_{z_{n-1}}^{\omega}, F_{z_{0}}^{\omega}, F_{z_{1}}^{\omega}$ are all contained in $F_{z_{0}}^{\nu}$. Because each $z_{i}$ lies outside $F_{z_{0}}^{\omega}, \ldots, F_{z_{i-1}}^{\omega}$, part (b) of Lemma 5.1 implies that $F_{z_{i}}^{\mu}$ is disjoint from $F_{z_{0}}^{\mu}, \ldots, F_{z_{i-1}}^{\mu}$. Thus

(h) The boxes $F_{z_{i}}^{\mu}$ are disjoint, $i=0, \ldots, n-1$.

Step 8 . We need to say a little more about the configuration of these boxes. Consider $F_{z_{i}}^{\omega}, F_{z_{i+1}}^{\omega}$. They lie in $F_{z_{i}}^{\nu}$ by construction. The part of $c F_{z_{i}}^{\nu}$ between $F_{z_{i}}^{\mu}$ and $F_{z_{i+1}}^{\mu}$ is a rectangle $R_{i}$, by (d). (If $i \neq n-1, R_{i}$ is contained in $F_{z_{i}}^{\omega}$, 
but for $i=n-1$, all we can say is that $R_{i}$ is part of the convex hull of $F_{z_{i}}^{\mu} \cup F_{z_{i+1}}^{\mu}$.) Suppose $F_{z_{j}}^{\mu}$ meets $R_{i}$. By (c) $\lambda \cap F_{z_{j}}^{\mu}$ is part of an arc stretching all the way across $F_{z_{i}}^{\nu}$. But $\lambda$ meets $F_{z_{i}}^{\nu}$ in a unique arc, namely the one containing $z_{i}$. Therefore $z_{j}$ lies on this arc. Since $F_{z_{j}}^{\mu}$ meets $R_{i}$, we see by (e) that $z_{j}$ lies between $z_{i}$ and $z_{i+1}$ on $\lambda$, a contradiction. Therefore,

(i) the rectangle $R_{i}$ which is the part of $c F_{z_{i}}^{\nu}$ between $F_{z_{i}}^{\mu}$ and $F_{z_{i+1}}^{\mu}$ meets no $F_{z_{j}}^{\mu}$. See Figure 5.9.

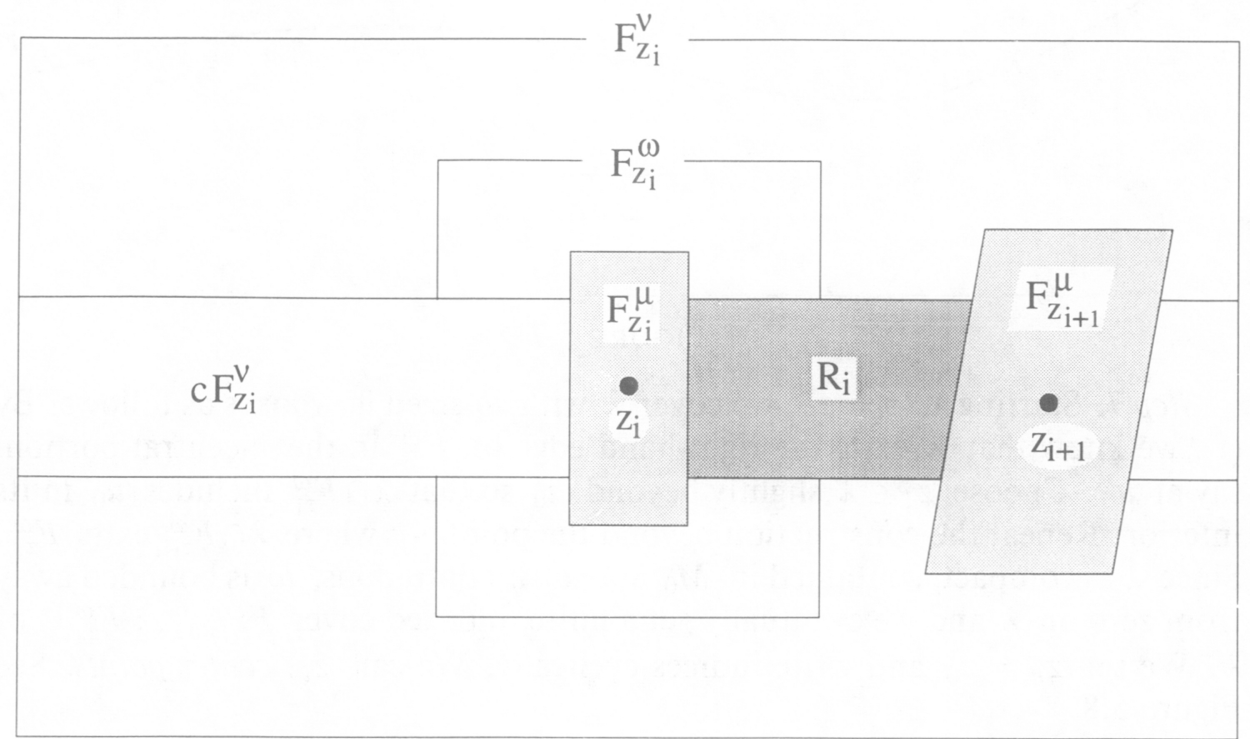

FIGURE 5.9

Step 9. Consider the point $v_{i}$ where $\lambda$ enters $F_{z_{i}}^{\mu}$. Let $y_{i}$ be the point of the left edge of $F_{z_{i}}^{\nu}$ on the $X$ trajectory through $v_{i}$. Thus, $\left[y_{i}, v_{i}\right]$ is a horizontal line in the $c$-central portion of $F_{z_{i}}^{\nu}$. Similarly, let $y_{i}^{\prime}$ be the point of the right edge of $F_{z_{i}}^{\nu}$, horizontally related to $v_{i+1}$. See Figure 5.10.

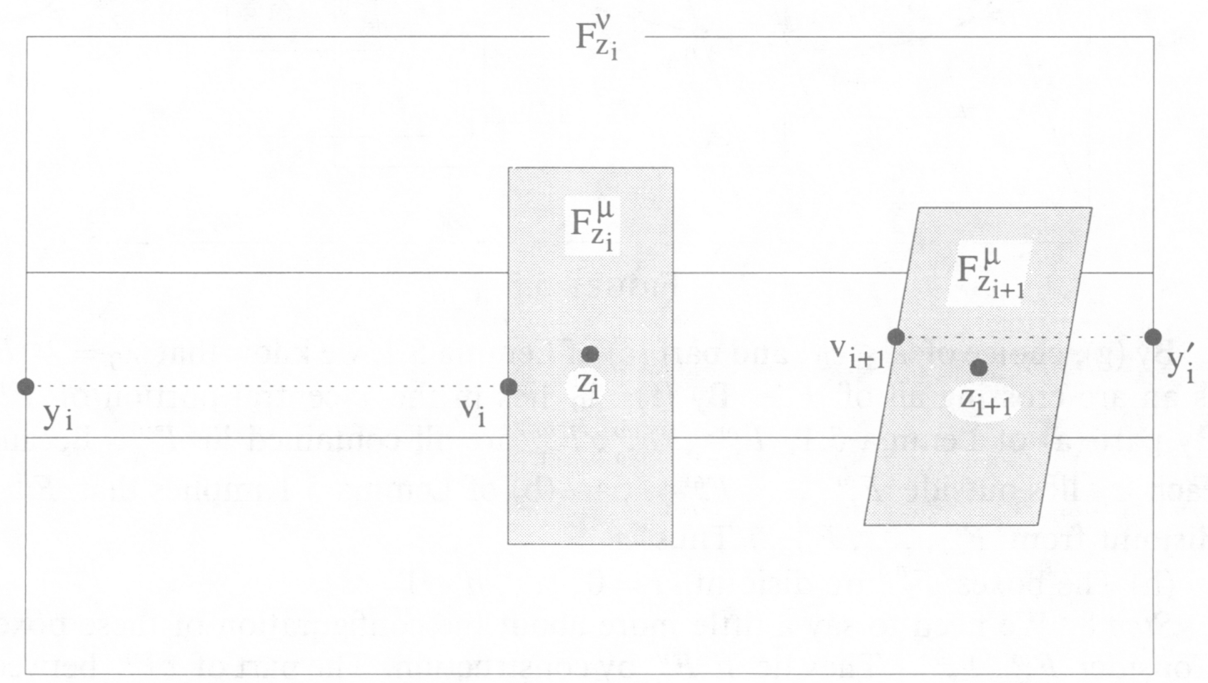

FIGURE 5.10 
By the Lift Lemma 3.1, there is a perturbation of $X, Y_{i}$, such that $Y_{i} \equiv X$ off $F_{z_{i}}^{\mu}$ and under the $Y_{i}$-flow we have

$$
y_{i} \rightarrow v_{i} \rightarrow z_{i} \rightarrow v_{i+1} \rightarrow y_{i}^{\prime} .
$$

For $y_{i}, y_{i}^{\prime}$ lie in the $c$-central portion of $F_{z_{i}}^{\nu}$. Let $\gamma_{i}$ be the part of this $Y_{i}$ trajectory from $v_{i}$ to $v_{i+1}$. It lies in the $c$-central portion of $F_{z_{i}}^{\nu}$ and therefore (j) $\gamma_{i} \subset\left(F_{z_{i}}^{\mu} \cup R_{i}\right)$. See Figure 5.11.

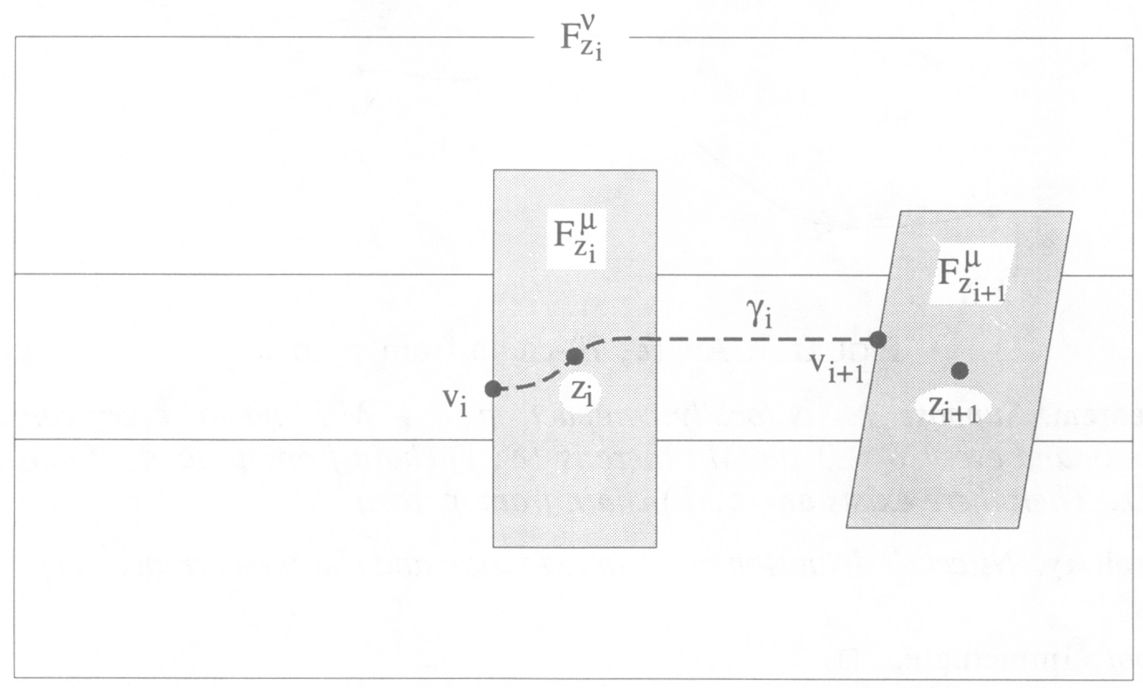

\section{FIGURE 5.11}

Step 10. Let $Y=X+\sum_{i=0}^{n-1}\left(Y_{i}-X\right)$. By the convexity property of the Whitney topology mentioned in Proposition 2.1 and disjointness of the supports of the $Y_{i}-X$ shown in (h), $Y \in \mathscr{U}$. We claim that $\gamma_{0} \cup \gamma_{1} \cup \cdots \cup \gamma_{n-1}$ is a periodic orbit of $Y$. By (i) none of the $Y_{j}$ affects the part of $\gamma_{i}$ in $R_{i}$ and, by disjointness of the supports $F_{z_{i}}^{\mu}$ of $Y_{i}-X$, none of the $F_{z_{j}}^{\mu}, j \neq i$, affects the part of $\gamma_{i}$ in $F_{z_{i}}^{\mu}$. Therefore $\gamma$ is indeed $Y$-periodic.

\section{APPENDIX 1}

Above and in Nitecki (1978) one uses $(\varepsilon, T)$-chains on noncompact manifolds with $\varepsilon$ a function but $T$ a constant. Here we show that the natural definition in which $T$ also is a function leads to the same chain extensions and chain limit sets.

Let $\varphi$ be a flow on the metric space $M$. An orbit segment of $\varphi$ is a set $\left\{\varphi_{t}(x) ; 0 \leq t \leq \tau\right\}$ which we denote by $[x, y], y=\varphi_{\tau}(x)$. An $(\varepsilon, T)$-chain from $p$ to $q$ is a finite union of orbit segments

$$
\Gamma=\bigcup_{i=1}^{n} \gamma_{i}, \quad \gamma_{i}=\left[x_{i}, y_{i}\right], y_{i}=\varphi_{t_{i}}\left(x_{i}\right),
$$

such that

(a) $t_{i} \geq T\left(x_{i}\right), 1 \leq i \leq n$

(b) $d\left(y_{i}, x_{i+1}\right)<\varepsilon\left(y_{i}\right), 0 \leq i \leq n$.

In (b) we understand $p=y_{0}$ and $q=x_{n+1}$. See Figure 1. The duration of $\gamma_{i}$ is $t_{i}$ and the duration of $\Gamma$ is $\sum t_{i}$. 


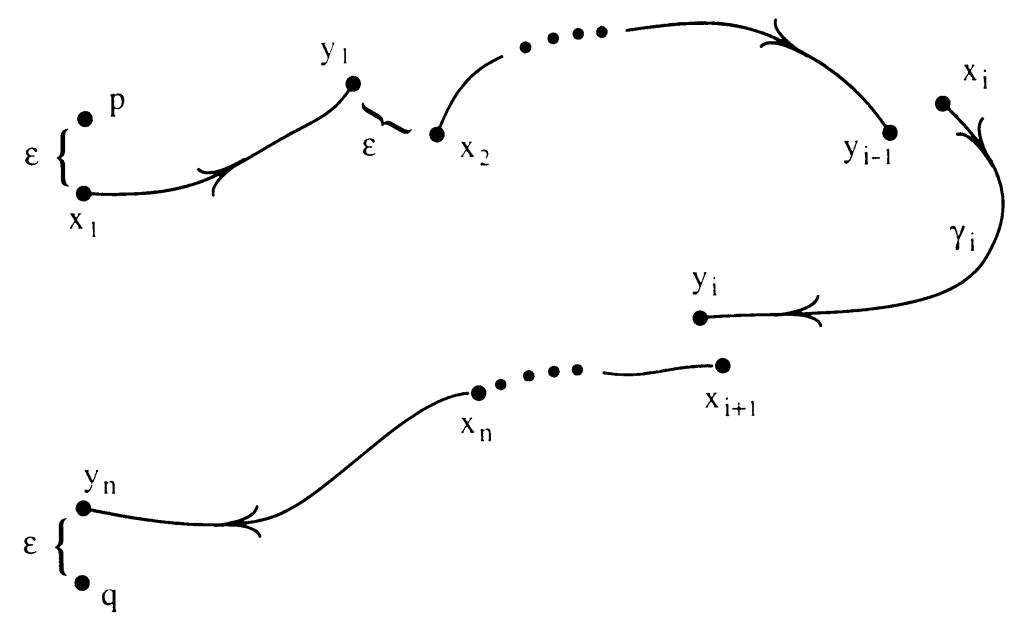

Figure 1. An $(\varepsilon, T)$-chain from $p$ to $q$.

Theorem. Suppose $M$ is locally compact, $p, q \in M$, and for every constant $D>0$ and every $\delta>0$ on $M$, there is $(\delta, 1)$-chain from $p$ to $q$ of duration $\geq D$. Then there exists an $(\varepsilon, T)$-chain from $p$ to $q$.

Corollary. Nitecki's definition of chain extension and chain recurrence agree with ours.

Proof. Immediate.

Proof of the theorem. Without loss of generality we assume $\varepsilon \leq 1$ and $T \geq 2$ on $M$. Also, we rescale the metric so all unit balls on $M$ are compact. Then we determine an appropriately small $\delta>0$ on $M$ as follows.

First, for eachi $y \in M$, we call

$$
\bar{T}(y)=3 \max \left\{T(z): d\left(z, \varphi_{t} y\right) \leq 1 \text { for some } t \in[0,2 T(y)]\right\} .
$$

In particular $\bar{T} \geq 3 T$. If $\delta>0$ is small and $\Gamma$ is a $(\delta, 1)$-chain from $y$ to $y^{\prime}$ of duration $\tau \leq \bar{T}(y)$ then

$$
d\left(\varphi_{\tau}(y), y^{\prime}\right) \leq \varepsilon\left(\varphi_{\tau} y\right) .
$$

Such a $\delta$ exists. For the set of such $\Gamma$ 's is compact and $y^{\prime} \rightarrow \varphi_{\tau}(y)$ as $\delta \rightarrow 0$. The convergence is uniform over all $\Gamma$ and all $\tau \in[0, \bar{T}(y)]$. That $\delta(y)$ can be chosen as a continuous positive function of $y \in M$ follows from continuity of $\varphi$ and local compactness of $M$.

By hypothesis, there is a $(\delta, 1)$-chain $\Gamma$ from $p$ to $q$ of duration $\geq \bar{T}(p)$. Break its orbit segments $\gamma_{1}, \ldots, \gamma_{n}$ into pieces, each of duration between 1 and 2. This introduces new jumps, but all of size zero. Thus, we assume, without loss of generality, that the orbit segments $\gamma_{i}=\left[x_{i}, y_{i}\right]$ of $\Gamma$ already have duration $t_{i} \in[1,2]$.

Successively choose $n_{1}<n_{2}<\cdots$ such that

$$
\begin{aligned}
T(p) & \leq T_{1}=t_{1}+\cdots+t_{n_{1}} \leq 2 T(p), \\
T\left(y_{n_{1}}\right) & \leq T_{2}=t_{n_{1}+1}+\cdots+t_{n_{2}} \leq 2 T\left(y_{n_{1}}\right),
\end{aligned}
$$

etc. Thus, the total duration of the first $n_{1}$ orbit segments is $T_{1} \doteq T(p)$, the total duration of the next $n_{2}-n_{1}$ is $T_{2} \doteq T\left(y_{n_{1}}\right)$, and so on. Since $T \geq 2$ 
and $t_{i} \in[1,2]$ it is always possible to choose $n_{1}<n_{2}<\cdots<n_{K} \leq n$ with these properties, $K \geq 1$ being the last choice. The final $n-n_{K}$ orbit segments have total duration $T_{K+1}<T\left(y_{n_{K}}\right)$ and so we combine them with the preceding $n_{K}-n_{K-1}$ durations as

$$
\tau_{K}=\sum_{n_{K-1}<i \leq n} t_{i}=T_{K}+T_{K+1} .
$$

When $k=1, \ldots, K-1$ we set $\tau_{k}=T_{k}$ and

$$
\begin{aligned}
\lambda_{1} & =\left\{\varphi_{t}(p): 0 \leq t \leq \tau_{1}\right\}=\left[p_{1}, z_{1}\right], \\
\vdots & \\
\lambda_{k} & =\left\{\varphi_{t}\left(y_{n_{k-1}}\right): 0 \leq t \leq \tau_{k}\right\}=\left[p_{k}, z_{k}\right], \\
\vdots & \\
\lambda_{K} & =\left\{\varphi_{t}\left(y_{n_{K-1}}\right): 0 \leq t \leq \tau_{K}\right\}=\left[p_{K}, z_{K}\right] .
\end{aligned}
$$

The chain $\Lambda=\lambda_{1} \cup \cdots \cup \lambda_{K}$ starts at $p_{1}=p$ and ends at $z_{K}$. The duration $\tau_{k}$ of $\lambda_{k}$ satisfies

$$
T\left(p_{k}\right) \leq \tau_{k} \leq 2 T\left(p_{k}\right)<\bar{T}\left(p_{k}\right), \quad 1 \leq k \leq K-1,
$$

by construction. For $k=K$ we have $p_{K}=y_{n_{K-1}}$ and

$$
\begin{aligned}
T\left(p_{K}\right) & =T\left(y_{n_{K-1}}\right) \leq T_{K}<T_{K}+T_{K+1} \\
& \leq 2 T\left(y_{n_{K-1}}\right)+T\left(y_{n_{K}}\right) \\
& \leq \frac{2}{3} \bar{T}\left(y_{n_{K-1}}\right)+\frac{1}{3} \bar{T}\left(y_{n_{K-1}}\right)=\bar{T}\left(p_{K}\right) .
\end{aligned}
$$

Thus, by $(*)$

$$
d\left(z_{k}, p_{k+1}\right) \leq \varepsilon\left(p_{k}\right), \quad 0 \leq k \leq K,
$$

where $z_{0}$ and $p_{K+1}$ denote $p$ and $q$. That is, $\Lambda$ is an $(\varepsilon, T)$-chain from $p$ to $q$ and the theorem is proved.

\section{APPENDIX 2}

It is not hard to see that the property of hyperbolicity of fixed points we used was:

(H) $p$ has a neighborhood $N$ such that every $\varepsilon$-quasiorbit in $N$ can be $\delta$-shadowed by a single $\varphi$-orbit-segment and $\delta \rightarrow 0$ uniformly as $\varepsilon \rightarrow 0$.

For conciseness we say $p$ is singly $\delta$-shadowable. It is easy to see that hyperbolic fixed points have property $\mathrm{H}$. So does the fixed point of

$$
\dot{x}=x^{2}+y^{2} \dot{y}=0 \text {. }
$$

See Figure 1. 


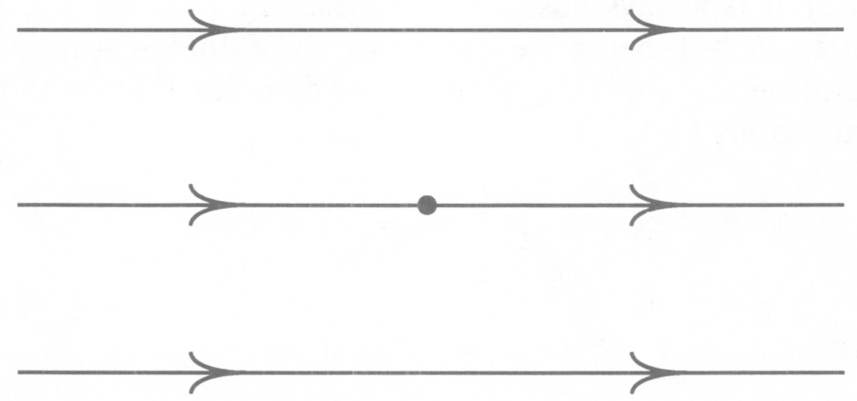

FIGURE 1

Proposition. Let $\varphi$ be a flow in the plane with an isolated fixed point $p$. Property $\mathrm{H}$ holds for $p$ if and only if either

(a) $\varphi$ is topologically conjugate to $(*)$ near $p$.

(b) $\varphi$ is topologically conjugate to a hyperbolic flow near $p$.

(c) $p$ is a rotation point of $\varphi$ with alternate inward-outward nonperiodic bands, no bands of closed orbits, and no semistable closed orbits.

Proof. In Hartman (1964) the local phase portrait of isolated planar fixed points is well described in terms of sectors.

First suppose $p$ is not a rotation point. Assume there exists an elliptic solution $E$. Inside $E$ is a second elliptic solution $E^{\prime}$. See Figure 2(a).
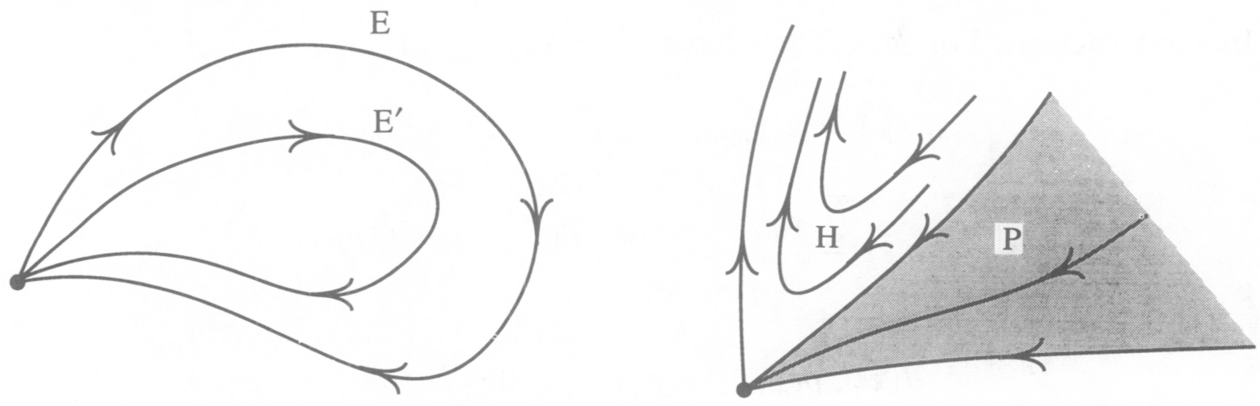

FIGURE 2

The $\varepsilon$-quisiorbit along $E$ followed by $E^{\prime}$ is not singly $\delta$-shadowable. Thus, there are no elliptic sectors at $p$. All the sectors are hyperbolic or parabolic, and none of them has any elliptic parts. They are pure.

If $p$ has a hyperbolic sector $H$ and also a parabolic sector $P$, say positive, then an $\varepsilon$-quasiorbit along an interior base-solution in $P$ joined to the negative base solution of $H$ by a $C^{1}$ curve cannot be singly $\delta$-shadowed. See Figure 2(b).

Therefore, if $p$ is not a rotation point then all its sectors are pure parabolic or all are pure hyperbolic. In the former case, $p$ is a source or sink. In the latter case the number $n_{h}$ of hyperbolic sectors is even because of the Poincare Index Formula (Hartman (1964)). If $n_{h}=2$ then $\varphi$ is topologically equivalent to $(*)$. If $n_{h}=4$, it is a hyperbolic saddle. If $n_{h} \geq 6$ then an $\varepsilon$-quasiorbit along a positive base-solution (separatrix) joined to a nonadjacent negative base solution by a $C^{1}$ curve is not singly $\delta$-shadowable. Consequently, $p$ obeys (a), (b), or is a rotation point, the case we now analyse. 
Rotation points $p$ are encircled by arbitrarily small closed orbits. Let $S$ be the set of all closed orbits near $p$. The set $S$ contains no annulus $A$ of closed orbits because there would exist $\varepsilon$-quasiorbits which are nearly dense in $A$. They would be singly unshadowable. Similarly an $\varepsilon$-quasiorbit along an ingoing spiral to a semistable closed orbit followed by an outgoing spiral (joined by a $C^{1}$ curve) is singly unshadowable.

Thus, the complement to $S \cup\{p\}$ near $p$ consists of a countable number of bands of nonperiodic orbits. By Poincaré Bendixson theory, all the points of such a band $B$ have the same $\omega$-limit set, one of the two boundary curves of $B$, and all the points have the other boundary curve as their $\alpha$-limit set. Say that $B$ is inward if $\omega(x)$ is inside $\alpha(x)$ for all $x \in B$ and outward if the opposite.

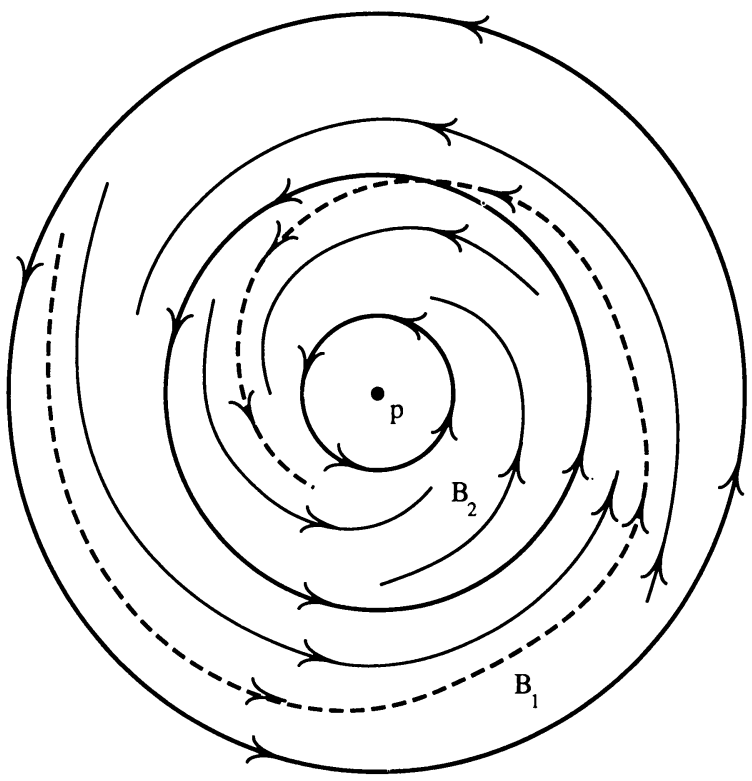

FIGURE 3

If between two inward bands $B_{1}$ and $B_{2}$ there are only inward bands then there exist $\varepsilon$-quasiorbits marching forever inward from $B_{1}$ through $B_{2}$ which are singly unshadowable. This is the semistable contradiction again.

Thus property $\mathrm{H}$ implies (a), (b), or (c). The converse is easily verified and left to the reader.

\section{REFERENCES}

N. P. Bhatia and G. P. Szegö, Stability theory of dynamical systems, Springer-Verlag, Berlin and New York, 1970.

Z. Nitecki, Explosions in completely unstable flows. I, Preventing explosions, Trans. Amer. Math. Soc. 245 (1978), 43-61.

M. L. A. Peixoto, The closing lemma for generalized recurrence in the plane, Trans. Amer. Math. Soc. 308 (1988).

C. C. Pugh, The closing lemma, Amer. J. Math. (2) 89 (1967), 956-1009.

An improved closing lemma and a general density theorem, Amer. J. Math. 89 (1967), 10101021. 
_, Against the $C^{2}$ closing lemma, J. Differential Equations 17 (1975), 435-443.

_, The $C^{1}$-connecting lemma, a counterexample (preprint), 1984.

Escola Nacional de Ciencias Estatisticas, Rua André Cavalcanti 106, 20231 Rio de JANEIRO, R.J., BRASIL

E-mail address: mpe@bribge.bitnet

Department of Mathematics, University of California, Berkeley, California 94720 